\title{
ATUAÇÃO DA REDE FEDERAL DE EDUCAÇÃO PROFISSIONAL CIENTÍFICA E TECNOLÓGICA QUANTO A OFERTA DE CURSOS TÉCNICOS EM SAÚDE
}

\section{PERFORMANCE OF THE FEDERAL FIELD OF PROFESSIONAL SCIENTIFIC AND TECHNOLOGICAL EDUCATION REGARDING THE OFFER OF TECHNICAL COURSES IN HEALTH}

\author{
RAMOS, Marise Nogueira ${ }^{1}$ \\ ANDRADE, Thayná Trindade da Silva de 2 \\ LIMA, Julio Cesar França ${ }^{3}$ \\ REIS, Gianne Cristina ${ }^{4}$
}

\begin{abstract}
RESUMO
Este artigo busca apresentar um panorama geral da oferta de cursos de educação profissional técnica em saúde pela Rede Federal de Educação Profissional, Cientifica e Tecnológica. Tratase de um estudo descritivo-exploratório em âmbito nacional, que analisa dados quantitativos da oferta de formação em saúde pela rede federal de 2010 a 2016, estabelecendo comparações com as demais esferas administrativas. Os temas analisados neste artigo, ainda que com resultados parciais, demonstram que a rede federal ampliou sua formação como um todo, em número de cursos e matrículas. No entanto, a formação técnica de nível médio em saúde ainda é ofertada hegemonicamente por instituições privadas. Os resultados mostram que a Rede Federal de Educação Profissional Cientifica e Tecnológica ofertou 4,8\% do total de cursos técnicos em saúde em 2016, tendo o maior quantitativo de matrículas concentrado em cursos de agente comunitário de saúde e análises clinicas.
\end{abstract}

Palavras-chave: Política de Educação Profissional e Tecnológica; Política de Educação Profissional em Saúde; Rede Federal de Educação Profissional, Científica e Tecnológica; Saúde Pública

\footnotetext{
${ }^{1}$ Fundação Oswaldo Cruz (FIOCRUZ) / Escola Politécnica de Saúde Joaquim Venâncio (EPSJV) / Laboratório de Trabalho e Educação Profissional em Saúde (LATEPS). Rio de Janeiro, RJ, Brasil. ORCID: 0000-0001-5439-3258 e-mail: marise.ramos@fiocruz.br

${ }^{2}$ Fundação Oswaldo Cruz (FIOCRUZ) / Laboratório de Trabalho e Educação Profissional em Saúde (LATEPS). Rio de Janeiro, RJ, Brasil. e-mail: thayna.t.andrade@gmail.com

${ }^{3}$ Fundação Oswaldo Cruz (FIOCRUZ) / Laboratório de Trabalho e Educação Profissional em Saúde (LATEPS). Rio de Janeiro, RJ, Brasil. e-mail: jcfflima@gmail.com

${ }^{4}$ Fundação Oswaldo Cruz (FIOCRUZ) / Escola Politécnica de Saúde Joaquim Venâncio (EPSJV) / Laboratório de Trabalho e Educação Profissional em Saúde (LATEPS). Rio de Janeiro, RJ, Brasil. e-mail: giannereis@gmail.com
} 
DOI: $10.12957 /$ e-mosaicos.2020.46666

\section{ABSTRACT}

This article seeks to present an overview of the offer of technical professional health education courses by the Federal Field of Professional, Scientific and Technological Education. This is a descriptive-exploratory study at a national level that analyzes quantitative data on the supply of technical training at the secondary level in health by the field from 2010 to 2016, comparing with the other administrative spheres. The themes analyzed in this article, although with partial results, show that the federal field has expanded its training as a whole, in terms of number of courses and enrollments, but the technical training of middle level in health is still offered hegemonically by the private institutions. The results show that the Federal Field of Professional Scientific and Technological Education offered $4.8 \%$ of the total number of technical courses in health in 2016, with the highest number of enrollments concentrated in courses of community health agent and clinical analysis

KeYwORDS: Professional and Technological Education Policy; Professional Education Policy in Health; Federal Network of Professional, Scientific and Technological Education; Public Health

\section{INTRODUÇÃO}

Este artigo apresenta resultados parciais da pesquisa $A$ Educação Profissional Técnica de Nível Médio em Saúde na rede federal de educação profissional e tecnológica brasileira, face ao atual Plano Nacional de Educação, que busca captar as concepções ético-políticas, epistemológicas e pedagógicas, que têm orientado a oferta de cursos técnicos de nível médio em saúde no âmbito federal. Neste momento, em que a rede federal de educação profissional e tecnológica torna-se um novo sujeito formador de trabalhadores técnicos da saúde, nosso problema de pesquisa visa observar essa oferta do ponto de vista quantitativo.

Para isso, analisamos a oferta quantitativa da educação profissional técnica de nível médio nas redes públicas de educação (federal, estadual, municipal), e na rede privada, no período 2010-2016. Buscamos revelar tendências e dinâmicas da relação público e privado de cursos da educação profissional em saúde, com foco na oferta de cursos pela rede federal. Procuramos oferecer um panorama geral do padrão de oferta de cursos e matrículas de nível médio tecnológico em saúde da rede federal de educação profissional científica e tecnológica, identificando e explicitando a possível ampliação da esfera pública neste campo, comparando oferta de cursos e matrículas da rede federal com as demais esferas administrativas.

Salientamos que identificar e explicitar a possível ampliação da esfera pública neste campo, bem como as esferas concorrentes, além de mostrar dimensões da realidade educacional no país, torna-se material concreto para a disputa pela política pública em coerência com a luta pela educação e pela saúde como direitos, no sentido de construir e defender uma rede integrada de atenção à saúde no SUS e uma política de educação integral, com ampla base para uma formação humana omnilateral. 
DOI: $10.12957 / \mathrm{e}-\mathrm{mosaicos} .2020 .46666$

\section{A REDE FEDERAL de EduCAÇÃo PROFissional E TECNOLógica E A FORMAÇÃo de TRABALHADORES TÉCNICOS EM SAÚDE}

As instituições da rede federal de educação profissional e tecnológica se caracterizaram historicamente, pela oferta de cursos para profissões industriais. A formação de trabalhadores técnicos de saúde, por sua vez, se realizou principalmente em escolas das redes municipais e estaduais, em escolas privadas - incluindo o SENAC - e nas ET-SUS 5 .

A partir das análises de Vieira e. al. (2013) podemos observar a dinâmica da formação técnica em saúde no Brasil na primeira década dos anos 2000. Em 1999, de acordo com o Censo educacional, a formação técnica de nível médio em saúde representava $11 \%$ do total de matrículas da educação profissional. Manifesta-se a predominância do setor privado (responsável por $62 \%$ da formação técnica de nível médio em saúde naquele ano ${ }^{6}{ }^{6}$, com aumento substantivo na década (em 2009 menos de um quarto - $24,7 \%$ - dos matriculados estavam em escolas técnicas públicas). As autoras reiteram o que foi constatado em estudos e pesquisas anteriores realizados por Pereira e Ramos (2006) e Ramos (2010), ou seja, a formação técnica em saúde no Brasil, como política pública - diferentemente da formação para a indústria, que foi preocupação do Estado desde o início do século - começa a tomar corpo nos anos de 1980, no contexto da Reforma Sanitária, com o Projeto Larga Escala. Tanto isto é verdade que o relatório da I Conferência Nacional de Recursos Humanos para a Saúde ressalta que o setor educacional - pela pequena oferta de vagas, pela inadequação curricular e por sua lógica privatizante - não vinha respondendo às necessidades de profissionalização em saúde. (Brasil/MS, 1986: 23)

Assim, diferentemente da formação para o setor primário da economia, as reformas privatizantes da educação profissional que ocorreram na década de 1990 não implicaram, na área da saúde, a necessidade de estrangulamento da instância pública. Ao contrário, foi suficiente não expandi-la e incentivar a ampliação da oferta privada. Desse modo, enquanto a matrícula nas escolas públicas que ofereciam formação técnica em saúde permaneceu abaixo de 40.000 alunos desde 1999 até 2005, as escolas privadas, que em 1999 já contavam com mais de 50.000 matriculados, passaram a contar, em 2005, com quase 200.000 alunos e continuaram crescendo significativamente mais do que o setor público até o final da década.

Outro fato destacado pelos autores é o significativo crescimento das matrículas na saúde, comparativamente aos outros setores. Enquanto esta quadruplicou entre 1999 e 2009, a matrícula total no mesmo período, torna-se cerca de uma vez e meia maior. Uma possível explicação para o fato por eles aventada, seria a correspondência com a criação mais acelerada de postos de trabalho no setor de serviços durante o período, com destaque além da educação, para a área de saúde.

\footnotetext{
${ }^{5}$ SENAC: Serviço Nacional de Aprendizagem Comercial. ET-SUS: Escolas Técnicas do Sistema Único de Saúde.

${ }^{6}$ Os autores mostram que, no total da educação profissional de nível técnico, naquele mesmo ano, 44\% das matrículas eram de responsabilidade do setor privado.
} 
DOI: $10.12957 /$ e-mosaicos.2020.46666

Estudo realizado por Frigotto et. al. (2015) demonstra que no Brasil, entre 2007 a 2012, os cursos de educação profissional mais oferecidos estão no eixo Ambiente e Saúde. Em coerência com a tendência demonstrada por Vieira et. al., enquanto em 2007 predominava a oferta privada de cursos neste eixo (20,9\% público e $79,1 \%$ privado), em 2012 verifica-se um aumento da oferta pública em todos os cursos técnicos - ainda que a relação não chegue a inverter - com exceção do eixo Informação e Comunicação. No eixo Ambiente e Saúde a oferta pública passa para $30,6 \%$ e a privada para $69,4 \%$.

Diante do exposto, torna-se uma questão de pesquisa saber que redes públicas (federal, estadual e municipal) têm contribuído para a expansão da oferta da educação profissional técnica de nível médio em geral e, particularmente, em saúde, no período de 2010 (ano em que observamos a inversão da oferta pela esfera pública) a 2016, tendo em vista as estratégias que preveem a integração da educação profissional à educação básica; e no caso da saúde, políticas e programas do Ministério da Saúde que redundam na ampliação da educação profissional em saúde.

\section{Metodologia}

Para iniciarmos este estudo, selecionamos os cursos do eixo Ambiente e Saúde como objeto, tendo como base o Catálogo Nacional de Cursos Técnicos ${ }^{7}$. Excluímos da análise os cursos da área ambiental ${ }^{8}$ e trabalhamos com 24 cursos estritamente da área de saúde, a saber: técnico em agente comunitário de saúde, técnico em análises clinicas, técnico em citopatologia, técnico em cuidador de idosos, técnico em enfermagem, técnico em equipamentos biomédicos, técnico em estética, técnico em farmácia, técnico em gerência em saúde, técnico em hemoterapia, técnico em imobilizações ortopédicas, técnico em imagem pessoal, técnico em massoterapia, técnico em necropsia, técnico em nutrição e dietética, técnico em óptica, técnico em órteses e próteses, técnico em podologia, técnico em prótese dentária, técnico em radiologia, técnico em reabilitação de dependentes químicos, técnico em registros e informações em saúde, técnico em saúde bucal e técnico em vigilância em saúde.

Após a seleção dos cursos, utilizamos como base de dados os microdados do Censo Escolar de 2010 a 2016, disponibilizados pelo Instituto Nacional de Estudos e Pesquisas Educacionais Anísio Teixeira (INEP). Após a seleção dos dados, utilizamos o software SPSS/IBM para filtragem dos dados e formatação das tabelas a serem analisadas.

Para composição das tabelas, selecionamos os dados referentes ao número de cursos e de matrículas de educação profissional em saúde ofertados no país de 2010 a 2016, na rede privada e pública, nos âmbitos federal, estadual e municipal. A partir

\footnotetext{
7 Catálogo Nacional de Cursos Técnicos (MEC, 2016).

${ }^{8}$ Os cursos excluídos da análise foram os cursos de Controle Ambiental, Meio Ambiente, Meteorologia e Reciclagem.
} 
DOI: $10.12957 / \mathrm{e}-\mathrm{mosaicos} .2020 .46666$

disso, filtramos os cursos e matrículas realizados estritamente pela Rede EPCT para análises e comparações.

\section{RESULTADOS E DISCUSSÃo}

Atualmente, trinta e oito Institutos Federais, dois Centros Federais de Educação Tecnológica (Cefets) e o Colégio Pedro II integram a Rede Federal de Educação Profissional, Científica e Tecnológica. Segundo dados da Plataforma Nilo Peçanha, referentes ao ano de 2018 (Plataforma Nilo Peçanha, 2019), 647 campi compõem a Rede, com uma oferta de 11.766 cursos e 45.486 professores. O gasto total no ano de 2018 foi de $R \$ 16.531 .953 .129,00^{9}$, um equivalente a $R \$ 15.725,66$ anuais por matrícula, R\$1.310,47 mensais por aluno. Segundo o Conselho Nacional das Instituições da Rede Federal de Educação Profissional, Científica e Tecnológica (CONIF), na proposta de Matriz Orçamentária da Rede Federal para o próximo ano, para suprir as necessidades da rede, serão necessários R $\$ 4,5$ bilhões (CONIF, 2019).

Dessas 647 unidades, 627 ofertam cursos técnicos, 215 ofertam cursos no eixo tecnológico ambiente e saúde ${ }^{10}$, representando $7,5 \%$ da oferta de cursos da Rede EPCT ${ }^{11}$ em 2018. Dentre esses, 77 são do subeixo tecnológico - saúde, composto pelos cursos por nós estudados. Entre essas unidades, apenas 10 ofertam cursos na modalidade integrado ao ensino médio.

Tabela 1: Número de Institutos Federais de Educação Profissional Científica e Tecnológica em 2018

\begin{tabular}{l|l}
\hline Rede EPCT & $\mathbf{6 4 7}$ \\
\hline Oferta de Cursos Técnicos & 627 \\
\hline Eixo Tecnológico Ambiente e Saúde & 215 \\
\hline Subeixo Tecnológico Saúde & 77 \\
\hline Cursos Técnicos Integrados & 10 \\
\hline \multicolumn{2}{|c|}{ Fonte: Elaborado pelos autores em julho de $2019^{12}}$.
\end{tabular}

Entre 2010 e 2016, 132 campi $^{13}$ da rede EPCT ofereceram pelo menos um curso do eixo estudado, distribuídos pelos estados brasileiros Acre, Alagoas, Espírito Santo, Mato Grosso, Rondônia e somente o estado de São Paulo não ofertava cursos do eixo da saúde pela Rede Federal de ensino. É possível inferir uma redução de campi que ofertam cursos neste segmento em comparação com os dados de 2018.

De acordo com os dados do INEP, em 2010 estavam sendo ofertados em nível nacional, 15.517 cursos de educação profissional e tecnológica. Em 2016, esse número

\footnotetext{
${ }^{9}$ Não estão incluídos no cálculo, os gastos das Escolas Técnicas vinculadas e a UNTPR.

10 O Curso técnico de Biotecnologia foi retirado da análise, porque até 2016 o curso encontrava-se no eixo técnico de produção industrial.

${ }^{11}$ Rede de Educação Profissional, Científica e Tecnológica.

12 Elaborado com base nos dados da Plataforma Nilo Peçanha (2018).

${ }^{13}$ Foram retirados da análise, os colégios técnicos vinculados a universidades e a UNTPR.
} 
DOI: $10.12957 / \mathrm{e}-\mathrm{mosaicos} .2020 .46666$

saltou para 32.947. Comparando 2010 a 2016, podemos observar um crescimento na oferta de cursos de 17.430; uma ampliação de $112 \%$.

Em relação ao número de matrículas, também observamos um crescimento, sendo que em 2010 havia 910.388 alunos matriculados em cursos da educação profissional e tecnológica e em 2016 esse quantitativo era de 1.672.491. Um aumento de 762.103 matriculados em relação a 2010; ou seja, houve um incremento de $84 \%$ no total de matrículas no período.

Quando seguimos a análise, na perspectiva dos cursos técnicos do subeixo da saúde, vemos que em 2016, em todas as esferas federativas, entre os cursos com maior número de oferta encontra-se o curso de Enfermagem (2454 cursos), seguido pelos cursos de Radiologia (463) e Análises Clínicas (398). O curso de Necropsia foi o menos ofertado no período em questão, com o total de (5 cursos).

Podemos observar uma ampliação da oferta em 1312 cursos comparando 2010 e 2016 no subeixo saúde. Uma importante observação pode ser realizada quanto ao curso de Análises Clínicas, o qual, pela série histórica, apresenta diminuição quantitativa de cursos, passando de 625 em 2010, para 398 em 2016. Embora este apresente redução da oferta de cursos, ainda permanece sendo um dos cursos mais ofertados no eixo saúde no período.

Quando voltamos a análise para a oferta de cursos na Rede Federal, podemos observar, de acordo com a figura 1 abaixo, que a referida rede, no ano de 2010, ofertava 93 cursos na área da saúde. Já no ano de 2016, esse quantitativo passou a ser de 253. Houve uma ampliação de 172\%; um aumento em números absolutos de 160 cursos, com pico de crescimento da oferta no ano de 2015 , com especial destaque para o curso de Agente Comunitário de Saúde, pois em 2014 eram ofertados 12 cursos sendo que em 2015 passaram a ser ofertados 42 e em 2016, 73. Assim, o curso de Agente Comunitário de Saúde de um dos menos ofertados pela Rede Federal ao posto de mais ofertado na rede Federal tornando a Rede em 2016 a maior ofertante de cursos de Agente comunitário de Saúde, uma ampliação na série histórica de $1360 \%$.

Figura 1: Total de Cursos Ofertados na esfera federal de educação profissional tecnológica em saúde entre os anos de 2010 e 2016

\section{Total de Cursos Ofertados}

Total de Cursos Ofertados

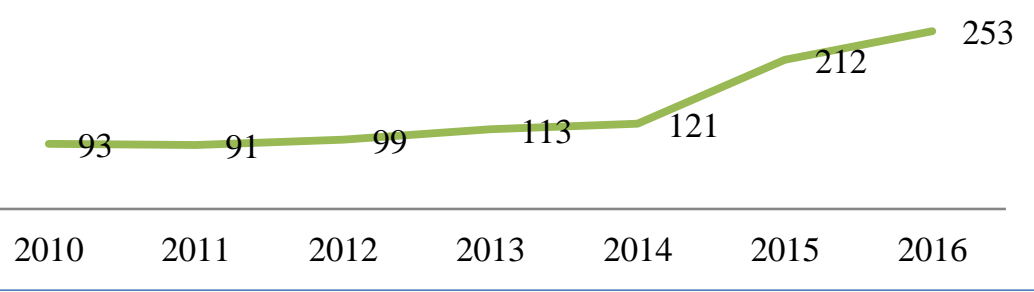

Fonte: Elaborado pelos autores em julho de 2019. 
DOI: $10.12957 /$ e-mosaicos.2020.46666

A Rede Federal de Educação Profissional Científica e Tecnológica - EPCT ofertou $4,8 \%$ do total de cursos técnicos em saúde em 2016. Considerando que em 2010 este número era de $2,3 \%$, houve uma ampliação de 2,5 pontos percentuais. No mesmo período, a ampliação de oferta de cursos técnicos em saúde nas demais esferas foi de $32,7 \%$, quando olhado somente o âmbito da rede federal, observamos que essa ampliação foi de $172 \%$ de cursos oferecidos em 2016 em relação a 2010.

Tabela 2: Cursos ofertados de Educação Profissional Tecnológica em Saúde pela Rede Federal entre os anos de 2010 e 2016

$\begin{array}{lllllll}2010 & 2011 & 2012 & 2013 & 2014 & 2015 & 2016\end{array}$

\begin{tabular}{|l|c|c|c|c|c|c|c|}
\hline Total Oferta & 4013 & 4065 & 4474 & 4694 & 5489 & 5448 & 5325 \\
\hline Rede Federal & 93 & 91 & 99 & 113 & 121 & 212 & 253 \\
\hline $\begin{array}{l}\text { \% de cursos ofertados } \\
\text { pela Rede Federal }\end{array}$ & 2,3 & 2,2 & 2,2 & 2,4 & 2,2 & 3,9 & 4,8 \\
\hline
\end{tabular}

Fonte: Elaborado pelos autores em julho de 2019.

Tabela 3: Comparativo de Matrículas em Cursos Técnicos da Educação Profissional

\begin{tabular}{|c|c|c|c|c|c|c|c|}
\hline $\begin{array}{c}\text { Matrículas Educação } \\
\text { Profissional }\end{array}$ & $\mathbf{2 0 1 0}$ & $\mathbf{2 0 1 1}$ & $\mathbf{2 0 1 2}$ & $\mathbf{2 0 1 3}$ & $\mathbf{2 0 1 4}$ & $\mathbf{2 0 1 5}$ & $\mathbf{2 0 1 6}$ \\
\hline $\begin{array}{c}\text { Matrículas Educação } \\
\text { Profissional em } \\
\text { Saúde }\end{array}$ & 243517 & 217743 & 206299 & 201558 & 285953 & 285505 & 345990 \\
\hline $\begin{array}{c}\text { \% de Matrículas em } \\
\text { Educação Profissional } \\
\text { em Saúde }\end{array}$ & 26,7 & 23,6 & 20,7 & 19,5 & 20,9 & 20,4 & 20,7 \\
\hline $\begin{array}{c}\text { Matrículas Educação } \\
\text { Profissional em } \\
\text { Saúde na Esfera } \\
\text { Federal }\end{array}$ & 5692 & 5089 & 5998 & 6246 & 6400 & 16863 & 14143 \\
\hline $\begin{array}{c}\text { \% de Matrículas na } \\
\text { Esfera Federal }\end{array}$ & 2,3 & 2,3 & 2,9 & 3,1 & 2,2 & 5,9 & 4,1 \\
\hline
\end{tabular}

Fonte: Elaborado pelos autores em julho de 2019.

Em relação ao número de matrículas, em 2010, 910.388 alunos estavam matriculados em cursos de educação profissional. Em 2016, esse número era de 1.672.491. Entre 2010 e 2016, o número de matrículas em cursos de educação profissional aumentou 762.103. Desse quantitativo, as matrículas em educação profissional em saúde foram de 102.473. Quando comparamos as matrículas em cursos de saúde com os dos outros eixos que não são saúde, observamos uma queda de 6.0 pontos percentuais nos cursos da saúde.

Em 2010, 26,7\% das matrículas dos cursos da educação profissional no Brasil eram da saúde. Em 2016, enquanto o número de matrículas totais da educação profissional ampliou em $83 \%$, o número de matrículas em saúde ampliou 42\%, o que 
DOI: $10.12957 /$ e-mosaicos.2020.46666

representou uma queda de $5 \%$ na proporção que representa as matrículas em saúde em relação às matrículas nas demais áreas no final deste período.

A Rede Federal apresentou $4,1 \%$ do total de matrículas em cursos profissionais de saúde em 2016. A Rede Privada é a que possui o maior quantitativo de matrículas, chegando a representar em 2016, 74,4\% das matrículas em cursos de saúde.

Tabela 4: Total de Matrículas em Cursos da Educação Profissional Tecnológica de Saúde por dependência administrativa entre os anos de 2010 e 2016

\begin{tabular}{c|c|c|c|c|c|c|c|c|c|} 
& Federal & \% & Estadual & \% & Municipal & \% & Privada & \% & Total \\
\hline $\mathbf{2 0 1 0}$ & 5692 & 2,0 & 79002 & 27,3 & 3236 & 1,12 & 201296 & 69,6 & 289226 \\
\hline $\mathbf{2 0 1 1}$ & 5089 & 1,8 & 61358 & 22,3 & 3072 & 1,11 & 206119 & 74,78 & 275638 \\
\hline $\mathbf{2 0 1 2}$ & 5998 & 2,1 & 70362 & 25,2 & 2773 & 0,99 & 200628 & 71,71 & 279761 \\
\hline $\mathbf{2 0 1 3}$ & 6246 & 2,2 & 63669 & 22,7 & 2866 & 1,02 & 207115 & 74 & 279896 \\
\hline $\mathbf{2 0 1 4}$ & 6400 & 1,7 & 66374 & 18,1 & 3626 & 0,99 & 290078 & 79,15 & 366478 \\
\hline $\mathbf{2 0 1 5}$ & 16863 & 4,7 & 65220 & 18,3 & 3881 & 1,09 & 270273 & 75,87 & 356237 \\
\hline $\mathbf{2 0 1 6}$ & 14143 & 4,1 & 70585 & 20,4 & 3870 & 1,12 & 257392 & 74,39 & 345999 \\
\hline
\end{tabular}

A variação no número de matrículas no período estudado foi de ampliação em quase todos os cursos, com exceção do Curso de Imagem Pessoal, Radiologia, Reabilitação de Dependentes Químicos e Registros e Informações em Saúde. Destacamos a ampliação de matrículas do curso de Agente Comunitário de Saúde (2088\%), de Vigilância em Saúde (545\%), e as de Análises Clínicas (323\%). Embora os cursos mais ofertados pela Rede Federal tenham sido o de Agente comunitário de saúde, seguido de Enfermagem e Análises Clínicas.

Comparando as redes federal, municipal, estadual e privada, no ano de 2016, a maior concentração de matrículas nos cursos de Agente Comunitário de Saúde (68\%), Equipamentos Biomédicos (57\%) e Vigilância em Saúde $(48,9 \%)$ esteve na rede federal de educação. É importante observar que entre 2010 e 2016, a rede federal passou de $2 \%$ do quantitativo total de matrículas em cursos de agente comunitário de saúde, para $68 \%$ do total de matrículas no ano.

Tabela 5 Matrículas em Cursos da Educação Profissional Tecnológica de Saúde por dependência administrativa entre os anos de 2010 e 2016

\begin{tabular}{|l|c|c|c|c|c|c|c|c|c|}
\hline & \multicolumn{10}{c|}{ por dependência administrativa entre os anos de 2010 e 2016} \\
\cline { 2 - 12 } & Federal & $\%$ & Estadual & $\%$ & Municipal & $\%$ & Privada & $\%$ & Total \\
\hline $\begin{array}{l}\text { Agente Comunitário } \\
\text { de Saúde }\end{array}$ & 4355 & 68 & 1643 & 26 & & 0,0 & 386 & 6 & 6384 \\
\hline Análises clínicas & 2041 & 11 & 7621 & 42 & 721 & 4,0 & 7865 & 43 & 18248 \\
\hline Citopatologia & & 0 & 29 & 10 & & 0,0 & 275 & 90 & 304 \\
\hline Enfermagem & 3364 & 2 & 36151 & 17 & 1181 & 0,6 & 169511 & 81 & 210207 \\
\hline $\begin{array}{l}\text { Equipamentos } \\
\text { Biomédicos }\end{array}$ & 254 & 57 & 12 & 3 & & 0,0 & 176 & 40 & 442 \\
\hline Estética & 141 & 1 & 1209 & 9 & & 0,0 & 11753 & 90 & 13103 \\
\hline
\end{tabular}


DOI: $10.12957 / \mathrm{e}-\mathrm{mosaicos} .2020 .46666$

\begin{tabular}{|l|c|c|c|c|c|c|c|c|c|}
\hline Farmácia & 383 & 4 & 2404 & 23 & 762 & 7,1 & 7123 & 67 & 10672 \\
\hline Gerência de Saúde & 337 & 18 & 816 & 44 & 251 & 13,5 & 453 & 24 & 1857 \\
\hline Hemoterapia & & 0 & 106 & 30 & 25 & 7,0 & 227 & 63 & 358 \\
\hline Saúde Bucal & 474 & 5 & 3051 & 34 & 556 & 6,2 & 4821 & 54 & 8902 \\
\hline Imagem Pessoal & & 0 & 60 & 25 & & 0,0 & 184 & 75 & 244 \\
\hline $\begin{array}{l}\text { Imobilizações } \\
\text { Ortopédicas }\end{array}$ & & 0 & 488 & 89 & & 0,0 & 60 & 11 & 548 \\
\hline Massoterapia & 197 & 5 & 683 & 18 & & 0,0 & 2890 & 77 & 3770 \\
\hline Nutrição e Dietética & 572 & 3 & 13547 & 68 & 84 & 0,4 & 5610 & 28 & 19813 \\
\hline Óptica & & 0 & & 0 & & 0,0 & 1723 & 100 & 1723 \\
\hline Órteses e Próteses & & 0 & 9 & 4 & 45 & 18,2 & 193 & 78 & 247 \\
\hline Podologia & & 0 & 120 & 5 & & 0,0 & 2385 & 95 & 2505 \\
\hline Prótese Dentária & 372 & 6 & 670 & 11 & 69 & 1,2 & 4731 & 81 & 5842 \\
\hline Radiologia & 297 & 1 & 810 & 2 & 20 & 0,1 & 36072 & 97 & 37199 \\
\hline $\begin{array}{l}\text { Reabilitação de } \\
\text { Dependentes } \\
\text { Químicos }\end{array}$ & 81 & 17 & 39 & 8 & & 0,0 & 363 & 75 & 483 \\
\hline $\begin{array}{l}\text { Registros e } \\
\text { Informações em } \\
\text { Saúde }\end{array}$ & 79 & 36 & 139 & 64 & & 0,0 & & 0 & 218 \\
\hline Vigilância em Saúde & 1038 & 49 & 831 & 39 & 156 & 7,4 & 96 & 5 & 2121 \\
\hline Cuidados de Idosos & 158 & 29 & 147 & 27 & & 0,0 & 233 & 43 & 538 \\
\hline Necropsia & & 0 & & 0 & & 0,0 & 262 & 100 & 262 \\
\hline Total & $\mathbf{1 4 1 4 3}$ & $\mathbf{7 0 5 8 5}$ & & $\mathbf{3 8 7 0}$ & & $\mathbf{2 5 7 3 9 2}$ & & \\
\hline
\end{tabular}

Fonte: Elaborado pelos autores em julho de 2019.

\section{CONCLUSÃo}

Os temas analisados neste artigo, ainda que com resultados preliminares, demonstram que a rede federal ampliou sua formação como um todo, seja em número de cursos ou de matrículas. No entanto, a formação técnica de nível médio em saúde ainda é ofertada hegemonicamente pela rede privada. Conforme observado, em 2016, $74 \%$ das matrículas em cursos de educação profissional em saúde concentravam-se na rede privada. Apesar de a Rede Federal de Educação Profissional Tecnológica ter tido um crescimento na sua oferta no período estudado, ainda representava um quantitativo baixo na oferta total de cursos da área de saúde (4,8\%) Mesmo observando um aumento da oferta pública de matrículas em todos os eixos, o aumento de matrículas no setor privado foi ainda maior (4\%).

A ampliação da oferta de cursos e de matrículas do curso de Agente Comunitário de Saúde (ACS) no período estudado se apresenta como um dado relevante na pesquisa, considerando que o Agente Comunitário de Saúde é um trabalhador fundamental no contexto da atenção básica, tendo sua inserção de trabalho fundamentalmente no setor público.

No que se refere à oferta de cursos de Vigilância em Saúde, novamente é possível observar que a rede tem direcionado sua formação para trabalhadores que 
têm tido sua inserção prioritária de trabalho no setor público. Analisamos esse processo como uma iniciativa importante na luta pela saúde pública do país e pela formação dos trabalhadores técnicos.

É importante salientar a ameaça dos postos de trabalho do Agente Comunitário de Saúde, com a Política de Atenção Básica (PNAB) de 2017, adotada pelo Ministério da Saúde, que reforçou a subtração de direitos e o desmonte do Sistema Único de Saúde (MOROSINI et. al., 2018). Na contramão das atuais políticas de supressão dos direitos, a Rede Federal tem realizado a formação para o trabalho de setores, cursos e áreas que não são lucrativas para o setor privado. No entanto, os cursos de Agente Comunitário de Saúde e de Agente de Combate a Endemias, por terem sua inserção profissional somente no setor público, tendem a ser menos procurados em virtude da baixa empregabilidade, ainda que sejam trabalhadores fundamentais dentro das equipes multiprofissionais da atenção básica.

Pode-se dizer que a ampliação significativa de matrículas na rede federal de 2014 para 2015, com pequena queda em 2016, é um movimento que coincide com a oferta de cursos efetivada através do PRONATEC - Programa Nacional de Apoio ao Ensino Técnico, implementado pelo Ministério da Educação em 2011 - naquele período, sendo necessárias pesquisas posteriores para avaliação dessa relação.

Este artigo, como salientado anteriormente, apresenta dados preliminares obtidos por meio das plataformas oficiais e trabalhados pelos autores. Devido ao volume de dados e o tempo necessário para obtenção e tratamento dos microdados fornecidos pela plataforma oficial do MEC, ainda não foi possível a análise de dados mais atuais, dos anos de 2017 a 2019, que são relevantes para o acompanhamento da formação dos trabalhadores técnicos da saúde em relação às novas medidas adotadas pelo governo, de cortes nos programas de formação de trabalhadores, como - PRONATEC, além das demais reformas, como a do Ensino médio, a Emenda Constitucional 95/2016, a adoção de políticas educacionais privatistas em detrimento da formação pública, que juntas formam um conjunto de reformas supressoras de direitos sociais como saúde, trabalho, previdência e educação.

Sob orientação do Artigo 7., da Lei no 11.892/2008, que estabelece que a Rede Federal deve ministrar cursos de educação profissional técnica de nível médio, prioritariamente na forma de cursos integrados, inclusive na modalidade EJA, salientamos a importância da produção de análises sobre esse tema no que se refere aos cursos de saúde, dado que a historicidade da educação profissional em saúde está ligada à formação de trabalhadores em serviço, o que se torna uma possível contradição entre o modelo preconizado pelos Institutos Federais e a necessidade de formação em saúde.

Tendo a Rede Federal em seu horizonte histórico a oferta de cursos com orientação mais industrial/tecnológica, estudos posteriores de análise qualitativa do modelo epistemológico que tem orientado essa oferta se fazem relevantes, na luta pela garantia de uma formação em saúde orientada para práticas integrais de saúde, alinhada aos princípios e diretrizes do Sistema Único de Saúde. 
DOI: $10.12957 /$ e-mosaicos.2020.46666

Portanto, inscrever o debate acerca das concepções e práticas das instituições que ofertam a educação profissional em saúde e suas contradições e associá-las à possibilidade de sua superação, na perspectiva de uma educação comprometida com a classe trabalhadora, nos parece uma condição fundamental para a consolidação do Sistema Único de Saúde e a construção de um tipo de sociedade cuja utopia sustentou as lutas da reforma sanitária.

\section{REFERÊNCIAS}

BRASIL. Ministério da Saúde. Secretaria Geral. Secretaria de Recursos Humanos. Conferência Nacional de Recursos Humanos para Saúde. Brasília: Ministério da Saúde, 1986. (Relatório Final) Disponível em: http://bvsms.

saude.gov.br/bvs/publicacoes/0116conf_rh.pdf. Acesso em: jun. 2012.

BRASIL, Ministério da Educação. Lei no 11.892 de 29 de dezembro de 2008, Institui a Rede Federal de Educação Profissional, Científica e Tecnológica, cria os Institutos Federais de Educação, Ciência e Tecnologia, e dá outras providências. Diário Oficial da República Federativa do Brasil, Brasília, 2008.

BRASIL, Ministério da Educação. Lei No 12.513, de 26 de outubro de 2011. Institui o Programa Nacional de Acesso ao Ensino Técnico e Emprego (PRONATEC). Diário Oficial da República Federativa do Brasil, Brasília, 2011.

BRASIL, emenda constitucional no 95, de 15 de dezembro de 2016. Altera o ato das disposições constitucionais transitórias, para instituir o novo regime fiscal, e dá outras providências. Brasília, 2016.

INEP. Censo da Educação Básica: 2010 a 2016. Brasília: Instituto Nacional de Estudos e Pesquisas Educacionais Anísio Teixeira.

FRIGOTTO, Gaudêncio et al. "Ofertas formativas e características regionais: a educação básica de nível médio no Estado do Rio de Janeiro". Relatório de Pesquisa. Edital 29/2012 Faperj - Apoio à Formação e Consolidação de Grupos de Pesquisa Multi-Institucionais e Interdisciplinares/2012. Rio de Janeiro, 2015.

Plataforma Nilo Peçanha. Acessado em 06 de julho de 2019. http://resultados.plataformanilopecanha.org/2019.

MOROSINI, Márcia Valéria Guimarães Cardoso, Fonseca, Angélica Ferreira e Lima, Luciana Dias de. Política Nacional de Atenção Básica 2017: retrocessos e riscos para o Sistema Único de Saúde. Saúde em Debate [online]. 2018, v. 42, n. 116, pp. 1124. Disponível em: https://doi.org/10.1590/0103-1104201811601. ISSN 2358-2898. https://doi.org/10.1590/0103-1104201811601. Acesso em 16 de julho 2019. 
DOI: $10.12957 /$ e-mosaicos.2020.46666

RAMOS, Marise N. Trabalho, educação e correntes pedagógicas no Brasil: um estudo a partir da formação dos trabalhadores técnicos da saúde. Rio de Janeiro: EPSJV, UFRJ 2010. 290 p.

RAMOS, Marise N.; PEREIRA, Isabel Brasil. Educação profissional em saúde. Rio de Janeiro: Editora Fiocruz, 2006.

Recebido em 14 de novembro de 2019

Aceito em 20 de maio de 2020

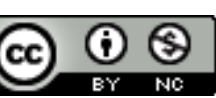

A e-Mosaicos Revista Multidisciplinar de Ensino, Pesquisa, Extensão e Cultura do Instituto de Aplicação Fernando Rodrigues da Silveira (CAp-UERJ) está licenciada com uma Licença Creative Commons - Atribuição-NãoComercial 4.0 Internacional.

Os direitos autorais de todos os trabalhos publicados na revista pertencem ao(s) seu(s) autor(es) e coautor(es), com o direito de primeira publicação cedido à e-Mosaicos.

Os artigos publicados são de acesso público, de uso gratuito, com atribuição de autoria obrigatória, para aplicações de finalidade educacional e não-comercial, de acordo com o modelo de licenciamento Creative Commons adotado pela revista. 